\title{
Acoso en el ambiente escolar: análisis de un cuestionario mediante Teoría de Respuesta al Ítem y Análisis de Correspondencias Múltiples*
}

\author{
Analysis of a School Bullying Questionnaire Using Item \\ Response Theory and Multiple Correspondence Analysis
}

Recibido: octubre 28 de 2011 |Revisado: diciembre 18 de 2012 |Aceptado: noviembre 26 de 2013

\author{
Víctor H. Cervantes Botero ** \\ EDIlBERTO CePEDA CUERVo**** \\ Martha Corrales Bossio *****
}

doi:10.11144/Javeriana.UPSY13-2.aaed

Para citar este artículo: Cervantes, V., Cepeda, E. \& Corrales, M. (2014). Acoso en el ambiente escolar: análisis de un cuestionario mediante Teoría de Respuesta al Ítem y Análisis de Correspondencias Múltiples. Universitas Psychologica, 13(2), 443-456. doi:10.11144/Javeriana.UPSY13-2.aaed

* Agradecimientos: Los autores agradecen a Cepeda C., Pacheco Durán, García Barco y Piraquive Peña (2008) (2008) por la facilitación de las bases de datos para la realización de este trabajo.

** Grupo de investigación Métodos e Instrumentos para la Investigación en Ciencias del Comportamiento, Universidad Nacional de Colombia, Bogotá. Correo electrónico: vhcervantesb@unal.edu.co

*** Departamento de Estadística, Universidad Nacional de Colombia, Bogotá. Correo electrónico: ecepedac@unal.edu.co

***** Departamento de Matemáticas, Universidad Sergio Arboleda, Bogotá. Correo electrónico: martha.corrales@usa.edu.co

\section{RESUMEN}

En este estudio se re-analizaron los datos de Cepeda C., Pacheco Durán, García Barco y Piraquive Peña (2008) mediante un modelo de Teoría de Respuesta al Ítem (TRI), y mediante Análisis de Correspondencias Múltiples (ACM) y de clasificación. Se encontró que las propiedades psicométricas del cuestionario de acoso escolar son bastante buenas ( $\alpha=0.96$ y buen ajuste al modelo unidimensional a partir del análisis paralelo). Además, se presentan las coincidencias entre los resultados de los dos tipos de análisis, y se encuentran resultados similares a otros estudios en Colombia. Los resultados de este estudio indican que el cuestionario analizado es una medida válida y confiable del acoso en el contexto escolar.

Palabras clave

Acoso escolar, evaluación del acoso, Teoría de Respuesta al Ítem, anclaje de la escala, Análisis de Correspondencias Múltiples, análisis de clasificación.

\footnotetext{
A B S T R A C T

This study presents reanalyzes of Cepeda et al. (2008)'s data using an Item Response Theory (IRT) model and Multiple Correspondence Analysis (MCA). Both internal consistency $(\alpha=0.96)$ and fit to the unidimensionality assumption (per parallel analysis) were found to be satisfactory. Coincidences between both analyses are shown. The percentage of students affected by bullying in the sample is similar to that found in other studies in Colombia. Finally, the questionnaire appears to be a valid and reliable measure of bullying.

Keywords

Bullying, bullying assessment, Item Response Theory, scale anchoring, Multiple Correspondence Analysis, cluster analysis.
} 
$\mathrm{El}$ acoso escolar representa un área de desarrollo e interés reciente en Colombia (v. g. Chaux, Bustamante, Castellanos, Jiménez \& Mejía, 2007; Chaux, Molano \& Podlesky, 2009; Cepeda et al., 2008; Jiménez, Castellanos \& Chaux, 2009) y en el mundo (v. g. Fleming \& Jacobsen, 2009, 2010; Hamburger, Basile \& Vivolo, 2011). Este se refiere al maltrato o agresión de la que es objeto un niño por parte de otro u otros estudiantes, de forma sistemática y repetida, en la cual no logra o no puede defenderse debido a un desbalance de poder (Olweus, 1993 citado por Jiménez et al., 2009; Oñate \& Piñuel, 2005).

Dado el interés y la mayor conciencia sobre los efectos negativos del acoso (cf. Jiménez et al., 2009), resulta importante examinar los métodos empleados para su evaluación y detección en el contexto escolar. Entre los procedimientos para recolectar información sobre el acoso se encuentran, por ejemplo, los utilizados por Fleming y Jacobsen $(2009,2010)$, quienes dieron una breve explicación escrita de lo que implica sufrir de acoso o intimidación escolar y preguntaron a los estudiantes si en el último mes habían sido intimidados. Chaux et al. (2009) emplearon una estrategia similar en la cual utilizaron un caso de ejemplo como medio de explicación del acoso y preguntaron a los estudiantes si se habían encontrado en alguno de los papeles de víctima, acosador o testigo de acoso en los últimos dos meses. Asimismo, se encuentran escalas para la evaluación del acoso escolar, tales como las desarrolladas por Mynard y Joseph (2000), Oñate y Piñuel (2005) o Cepeda et al. (2008); en este sentido, el interés en esta forma de evaluación del acoso escolar se refleja en una reciente com- pilación de las escalas desarrolladas en inglés sobre el mismo (Hamburger et al., 2011) o una reciente revisión de las propiedades psicométricas de varias escalas de maltrato (Rodríguez \& Martínez, 2009). En general, los reportes psicométricos sobre estos instrumentos consisten en la evaluación de su confiabilidad, para ninguno de los instrumentos se reportan coeficientes $\alpha$ inferiores a 0.7 y para algunos de ellos medidas de validez convergente. Es importante anotar que todos los instrumentos reseñados en Hamburger et al. (2011) y en Rodríguez y Martínez (2009) son cuestionarios en inglés y que para solo dos se reportan análisis de validez de constructo; de estos el cuestionario de maltratador/víctima de Olweus (OVBQ) ha sido analizado mediante modelos de Teoría de Respuesta al Ítem (Kyriakides, Kaloyirou \& Lindsay, 2006).

El objetivo de este estudio fue buscar una descripción más detallada de la calidad psicométrica del cuestionario de acoso escolar modificado por Cepeda et al. (2008) a partir de la utilización de métodos de Teoría de Respuesta al Ítem (TRI) y del Análisis de Correspondencias Múltiples (ACM). Como señalan Rodríguez y Martínez (2009), el acoso es una "conducta que se puede observar (...) y que como característica conductual implica información sobre contexto, frecuencia, intensidad e incluso sobre la forma como se presenta (pp. 133-134)"; esta particularidad hace especialmente importante evaluar el uso de una única puntuación obtenida mediante un cuestionario, es decir, obtener evidencias sobre su validez de constructo, en comparación con una descripción multivariada como la que se puede obtener mediante un ACM y un análisis de clasificación.

\section{TABLA 1}

Distribución por grado escolar y sexo

\begin{tabular}{lccccccc}
\hline & \multicolumn{7}{c}{ Grado } \\
\cline { 2 - 8 } \multicolumn{1}{c}{ Sexo } & $\mathbf{6}$ & $\mathbf{7}$ & $\mathbf{8}$ & $\mathbf{9}$ & $\mathbf{1 0}$ & $\mathbf{1 1}$ & Total \\
\hline Femenino & 358 & 288 & 265 & 225 & 202 & 414 & 1752 \\
Masculino & 386 & 201 & 225 & 181 & 205 & 276 & 1474 \\
Total & 744 & 489 & 490 & 406 & 407 & 690 & 3226 \\
\hline
\end{tabular}

Fuente: Adaptada de la Tabla 1 de Cepeda et al. (2008). 


\section{Método}

\section{Participantes}

El cuestionario de acoso escolar fue aplicado por Cepeda et al. (2008) a 3.226 estudiantes entre 10 y 20 años de edad $(\mathrm{M}=14.23, \mathrm{DE}=2.1)$ provenientes de instituciones educativas oficiales de la localidad Simón Bolívar en Bogotá. En la Tabla 1 se presenta la distribución de los estudiantes de acuerdo con el grado escolar cursado y el sexo del estudiante.

\section{Instrumento}

\section{Cuestionario de acoso escolar}

Este cuestionario fue elaborado por Cepeda et al. (2008), quienes adaptaron al contexto colombiano la escala Cisneros de acoso escolar presentada por Oñate y Piñuel (2005). El propósito de este instrumento es indagar sobre algunas formas de acoso físico o psicológico que pueden presentarse entre los estudiantes de educación básica secundaria. Está compuesto por 22 ítems con las siguientes opciones de respuesta: Nunca, A veces y Con frecuencia; los ítems que componen la escala se pueden consultar en el Apéndice. El coeficiente $\alpha$ de Cronbach fue de 0.96.

\section{Procedimiento}

La información recogida por Cepeda et al. (2008) fue procesada empleando el software R (R Development Core Team, 2008). Se produjeron nuevos análisis de los datos sobre la estructura del cuestionario para producir información sobre su validez de constructo.

\section{Análisis de datos}

\section{Análisis TRI}

El modelo TRI ajustado a los datos fue el modelo de respuesta graduada de Samejima ([GRM]; Samejima, 1969) empleando el paquete ltm (Rizo- poulos, 2006). Previamente a su ajuste, se examinaron a) el supuesto de unidimensionalidad del cuestionario de acoso escolar, a partir del análisis paralelo de la matriz de correlaciones policóricas entre los ítems (Cho, Li \& Bandalos, 2009; Glorfeld, 1995) y b) el supuesto de odds proporcionales respecto a las opciones de respuesta, a partir de la comparación entre los resultados del modelo GRM y de los modelos unidimensionales de dos parámetros a los que se reduce el modelo cuando se combinan dos de las tres categorías de los ítems y que representan los modelos acumulados hasta distintas categorías. De acuerdo con el supuesto de odds proporcionales del modelo GRM, las discriminaciones estimadas en los tres casos debieran mostrar una relación lineal.

Posteriormente, dada la escala definida por los parámetros de los ítems estimados para el GRM, se realizó una descripción de la misma mediante el procedimiento de anclaje de la escala (Beaton \& Allen, 1992; Cervantes, Cepeda C. \& Camargo, 2008) y se exploró la relación entre el cuestionario de acoso escolar con la edad, el sexo, el estrato socioeconómico, el grado cursado y el número de personas con las que convive en el hogar mediante un modelo de regresión latente (Antal, 2007).

\section{Análisis ACM y de clasificación}

El ACM fue ajustado por medio de las librerías ade4 (Chessel, Dufour \& Thioulouse, 2004) y FactoMineR (Husson, Josse, Le \& Mazet, 2007), y se realizó empleando como variables activas las respuestas al cuestionario y como variables ilustrativas la edad, el sexo, el estrato socioeconómico, el grado cursado y el número de personas con las que convive en el hogar. Los individuos participantes fueron caracterizados de acuerdo con el resultado del ACM. para lo cual se utilizaron los primeros ejes factoriales, seleccionados de acuerdo con el criterio de Barbary (1996), en un análisis de clasificación jerárquica, empleando el método de Ward, cuya solución fue optimizada mediante el algoritmo de k-medias (Lebart, Piron \& Morineau, 1995). 


\section{Resultados}

La Tabla 2 presenta el promedio y la desviación estándar de la edad de los participantes por grado cursado. La Tabla 3 muestra la distribución del número de personas con las cuales conviven los participantes de acuerdo con su sexo y el grado que cursaban en el momento de la aplicación. La Tabla 4 registra la distribución por estrato y grado cursado.

\section{Modelo de Respuesta al Ítem}

Evaluación de la unidimensionalidad de la escala

La Figura 1 presenta el gráfico de sedimentación de los valores propios asociados a la matriz de correlaciones policóricas junto con el percentil 95 para cada valor propio de acuerdo con el procedimiento de análisis paralelo.

TABLA 2

Media y desviación estándar de la edad de los estudiantes por grado escolar

\begin{tabular}{rcccccc}
\hline Grado & 6 & 7 & 8 & 9 & 10 & 11 \\
\hline Media (años) & $12.3(1.3)$ & $12.9(1.3)$ & $13.7(1.2)$ & $14.6(1.3)$ & $15.8(1.1)$ & $16.7(1)$ \\
\hline
\end{tabular}

Fuente: Reproducida de la Tabla 5 de Cepeda et al. (2008).

TABLA 3

Distribución de número de personas con quien convive por grado escolar y sexo

\begin{tabular}{|c|c|c|c|c|c|c|c|c|c|c|c|c|c|}
\hline \multirow{2}{*}{ \# Conv. } & \multicolumn{6}{|c|}{ Femenino } & \multicolumn{6}{|c|}{ Masculino } & \multirow[b]{2}{*}{ Total } \\
\hline & 6 & 7 & 8 & 9 & 10 & 11 & 6 & 7 & 8 & 9 & 10 & 11 & \\
\hline 1 & 3 & 2 & 1 & 2 & 2 & 3 & 3 & 1 & 2 & 1 & 1 & 1 & 22 \\
\hline 2 & 7 & 5 & 8 & 3 & 1 & 14 & 15 & 7 & 5 & 3 & 5 & 12 & 85 \\
\hline 3 & 19 & 9 & 2 & 8 & 7 & 10 & 30 & 6 & 7 & 8 & 6 & 15 & 127 \\
\hline 4 & 4 & 4 & 6 & 4 & 2 & 16 & 5 & 3 & 3 & 8 & 3 & 1 & 59 \\
\hline 5 & 170 & 133 & 132 & 96 & 79 & 169 & 164 & 96 & 92 & 78 & 88 & 138 & 1435 \\
\hline 6 & 76 & 54 & 63 & 36 & 44 & 74 & 112 & 45 & 62 & 44 & 50 & 49 & 709 \\
\hline 7 & 8 & 4 & 7 & 8 & 4 & 3 & 4 & 6 & 12 & 4 & 5 & 13 & 78 \\
\hline 8 & 69 & 72 & 41 & 64 & 62 & 121 & 53 & 36 & 41 & 34 & 42 & 43 & 678 \\
\hline 9 & 2 & 5 & 5 & 4 & 1 & 4 & 0 & 1 & 1 & 1 & 5 & 4 & 33 \\
\hline Total & 358 & 288 & 265 & 225 & 202 & 414 & 386 & 201 & 225 & 181 & 205 & 276 & 3226 \\
\hline
\end{tabular}

Fuente: elaboración propia

TABLA 4

Distribución por grado escolar y estrato

\begin{tabular}{cccccc}
\hline Grado & \multicolumn{5}{c}{ Estrato } \\
\cline { 2 - 5 } Cursado & 0 & 1 & 2 & 3 & NA \\
\hline 6 & & 505 & 224 & 13 & 2 \\
7 & & 347 & 130 & 12 & \\
8 & & 353 & 128 & 9 & \\
9 & 2 & 305 & 93 & 8 & \\
10 & & 199 & 202 & 4 & \\
11 & 2 & 452 & 221 & 17 & 2 \\
Total & 2161 & 998 & 63 & \\
\hline
\end{tabular}

Fuente: elaboración propia 


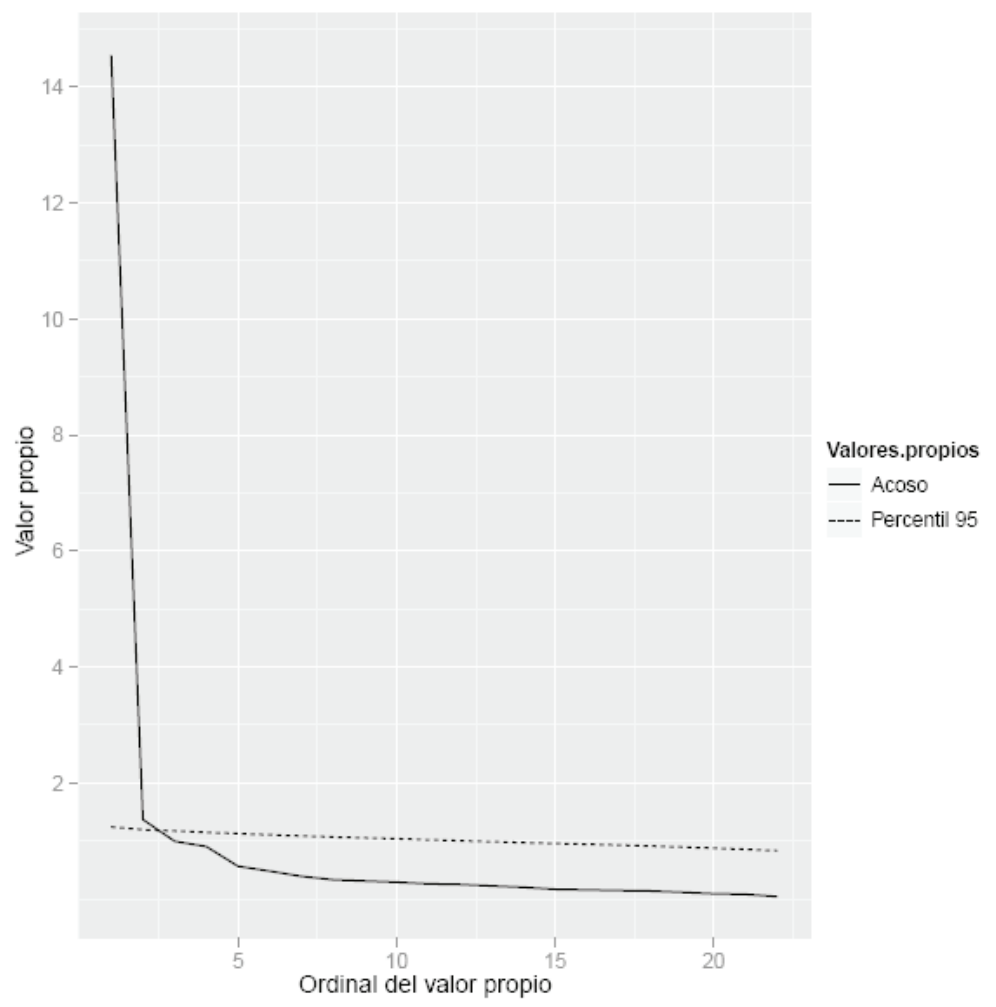

Figura 1. Gráfico de sedimentación de los valores propios.

Fuente: elaboración propia

El análisis indica que los 22 ítems del cuestionario presentan una estructura unidimensional, pues si bien tanto el primero como el segundo de los valores propios superan el percentil 95, la razón entre entre ellos es alta (10.64), la solución de un solo factor recoge el $64 \%$ de la varianza, mientras que el segundo permite recoger solamente un 5\% adicional, y la solución de dos factores muestra que todos los ítems pesan positivamente en ambos en una relación aproximadamente lineal ${ }^{1}$. En la Tabla 5 se muestra la matriz de pesos factoriales de la extracción por máxima verosimilitud del primer factor y la comunalidad para cada ítem bajo esta solución; se aprecia que los ítems 4 y 5 de la escala no se encuentran tan bien representados como los demás por el factor extraído.

1 Esta solución no se presenta.

\section{Exploración supuesto de odds proporcionales}

Para explorar el supuesto de odds proporcionales, se compararon las discriminaciones de los ítems estimadas por GRM y por los modelos acumulados por las distintas dicotomizaciones posibles. La Figura 2 presenta los gráficos de dispersión entre las discriminaciones estimadas de los ítems; los ejes representan las discriminaciones de acuerdo con su tratamiento como respuesta completamente ordinal (ORD) bajo el modelo GRM o alguna de las dos posibles dicotomizaciones en respuestas acumuladas, D1 al combinar las categorías 1 y 2, o D2 al combinar las categorías 2 y 3; así, la subfigura en la segunda fila y tercera columna representa en el eje $\mathrm{X}$ las discriminaciones para el análisis ORD y el eje Y las correspondientes a la dicotomización D2; las subfiguras en la diagonal muestran la distribución de las estimaciones en cada caso. Salvo por una estimación atípica para la discriminación del ítem 10 bajo la dicotomización que se obtiene 
TABLA 5

Comunalidad y pesos factoriales

\begin{tabular}{ccc}
\hline Ítem & Comunalidad & Peso \\
\hline AC1 & 0.701 & 0.837 \\
AC2 & 0.707 & 0.841 \\
AC3 & 0.707 & 0.841 \\
AC4 & 0.235 & 0.485 \\
AC5 & 0.374 & 0.612 \\
AC6 & 0.723 & 0.85 \\
AC7 & 0.816 & 0.903 \\
AC8 & 0.566 & 0.752 \\
AC9 & 0.792 & 0.89 \\
AC10 & 0.786 & 0.887 \\
AC11 & 0.698 & 0.835 \\
AC12 & 0.783 & 0.885 \\
AC13 & 0.674 & 0.821 \\
AC14 & 0.661 & 0.813 \\
AC15 & 0.811 & 0.9 \\
AC16 & 0.581 & 0.762 \\
AC17 & 0.582 & 0.763 \\
AC18 & 0.6 & 0.775 \\
AC19 & 0.586 & 0.766 \\
AC20 & 0.7 & 0.836 \\
AC21 & 0.548 & 0.74 \\
AC22 & 0.585 & 0.765 \\
\hline Varianza explicada & & 0.642 \\
\hline
\end{tabular}

Fuente: elaboración propia

de la combinación de las categorías 2 y 3 (D2), la relación entre las discriminaciones parece apoyar que el supuesto de odds proporcionales se cumple.

Ajuste del modelo TRI de respuesta graduada

La Tabla 6 presenta los parámetros del GRM estimados para los 22 ítems de la escala; la indeterminación de la escala asociada con los modelos TRI se eliminó al fijar la discriminación del primer ítem en 1 y uno de sus umbrales en 0 . Así como se observó en la Tabla 5 , los ítems 4 y 5 presentan discriminaciones bastante inferiores en comparación con los demás. Teniendo esto en cuenta, estos dos ítems no se incluyeron en el análisis de anclaje de la escala y podrían ser eliminados del cuestionario. Sin embargo, el coeficiente $\alpha$ de Cronbach solo se incrementa de 0.958 a 0.961 si se eliminan estos dos ítems.
TABLA 6

Parámetros de los ítems bajo el GRM

\begin{tabular}{cccc}
\hline Ítem & Umbral 1 & Umbral 2 & Discriminación \\
\hline AC1 & -2.262 & 0 & 1 \\
AC2 & -0.822 & 1.656 & 0.921 \\
AC3 & -2.082 & 0.237 & 1.003 \\
AC4 & -2.602 & 2.676 & 0.383 \\
AC5 & -1.399 & 2.724 & 0.558 \\
AC6 & -3.226 & 0.114 & 1.163 \\
AC7 & -2.069 & 0.156 & 1.404 \\
AC8 & -2.842 & 0.316 & 0.783 \\
AC9 & -2.647 & -0.354 & 1.377 \\
AC10 & -2.15 & 0.712 & 1.249 \\
AC11 & -3.163 & 0.293 & 1.099 \\
AC12 & -2.127 & 0.461 & 1.248 \\
AC13 & -1.863 & 0.838 & 0.975 \\
AC14 & -2.75 & 0.399 & 0.925 \\
AC15 & -2.033 & 0.506 & 1.336 \\
AC16 & -2.3 & 1.656 & 0.807 \\
AC17 & -2.174 & 0.784 & 0.815 \\
AC18 & -2.883 & 0.744 & 0.823 \\
AC19 & -1.694 & 2.338 & 0.793 \\
AC20 & -0.417 & 1.392 & 0.854 \\
AC21 & -1.694 & 1.446 & 0.699 \\
AC22 & -3.084 & -0.591 & 0.85 \\
\hline
\end{tabular}

Fuente: elaboración propia

\section{Anclaje de la escala}

Siguiendo el procedimiento presentado por Cervantes et al. (2008), se encontraron empíricamente los valores $-3.812,-0.81,1.544$ y 3.118 como los más apropiados para describir la escala de acoso escolar. La Figura 3 presenta los intervalos asociados con cada umbral de los ítems del cuestionario bajo el GRM que representan los cambios en la magnitud de acoso percibido con la probabilidad de dar cierta respuesta a cada uno de los ítems. De acuerdo con los valores encontrados se pueden definir los siguientes niveles en la escala de acoso:

Nivel 0: Hasta un valor de acoso estimado de -3.812. Reporta que Nunca le ocurren situaciones asociadas con cualquiera de los ítems.

Nivel 1: Entre un valor de acoso estimado de -3.812 y -0.81 . Empieza a reportar que $A$ veces le 

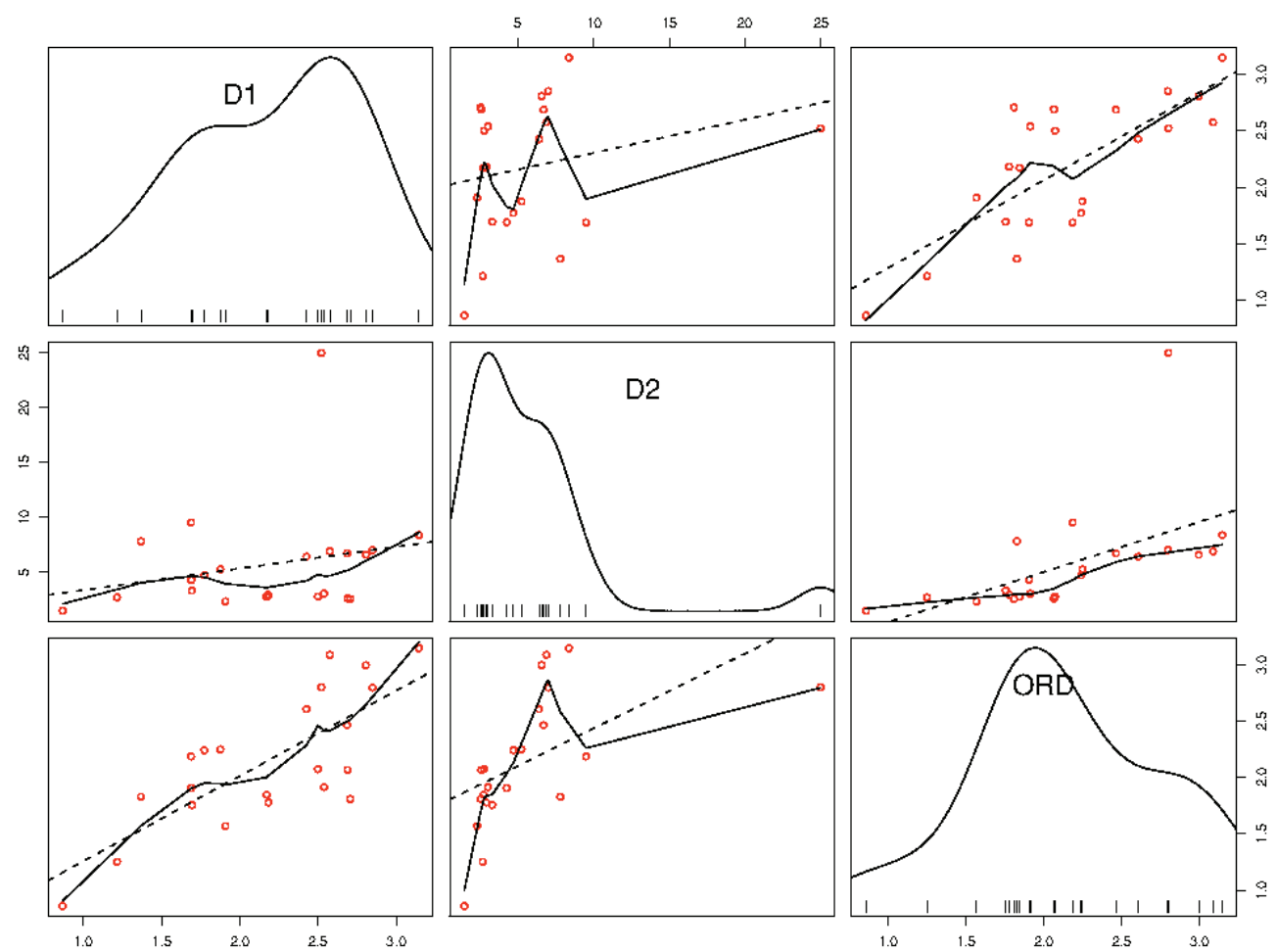

Figura 2. Parámetros de discriminación de los ítems para las dos posibles dicotomizaciones (D1 y D2) y para el GRM (ORD).

Fuente: elaboración propia

ocurren la mayoría de situaciones descritas por los ítems del cuestionario. Cuando el acoso estimado es más cercano a -0.81 , es muy probable que reporte que Con frecuencia le ocurra que le digan apodos que no le gusten (ítem 22). En general, en este rango es muy probable que aún reporte que Nunca le quiten las onces (ítem 20), ni le obliguen a hacer cosas que pongan en peligro su salud (ítem 2).

Nivel 2: Valores estimados de acoso entre -0.81 y 1.544. Empieza a reportar que Con frecuencia le ocurren muchas de las situaciones descritas por los ítems. Solamente cuando el acoso estimado es cercano a 1.544 se observa que la probabilidad de reportar que Con frecuencia le obliguen a hacer cosas que pongan en peligro su salud (ítem 2), le hagan bromas crueles sobre su aspecto físico (ítem 16), le quiten las onces (ítem 20) o le induzcan a actuar violentamente (ítem 21) se acerca a 0.5. Además, es muy poco probable que reporte que Con frecuencia dañen sus objetos personales (ítem 19).
Nivel 3: Valores estimados de acoso entre 1.544 y 3.118. Empieza a ser muy probable que Con frecuencia le obliguen a hacer cosas que pongan en peligro su salud (item 2), le hagan bromas crueles sobre su aspecto físico (ítem 16), le quiten las onces (ítem 20) o le induzcan a actuar violentamente (ítem 21).

Nivel 4: Valores de acoso estimado mayores a 3.118. Reporta que Con frecuencia le ocurren situaciones asociadas con todos los ítems.

Ajuste de los modelos de regresión latente

Los modelos de regresión latente utilizados toman como conocidos los parámetros de los ítems del GRM presentados en la sección anterior y plantean un modelo lineal para la variable latente que representa al acoso escolar en el modelo TRI (Aitkin \& Aitkin, 2011; Antal, 2007). Se ajustaron dos modelos de regresión latente en un modelo lineal mixto generalizado; en el primero ( $\mathrm{MN}$ o modelo 


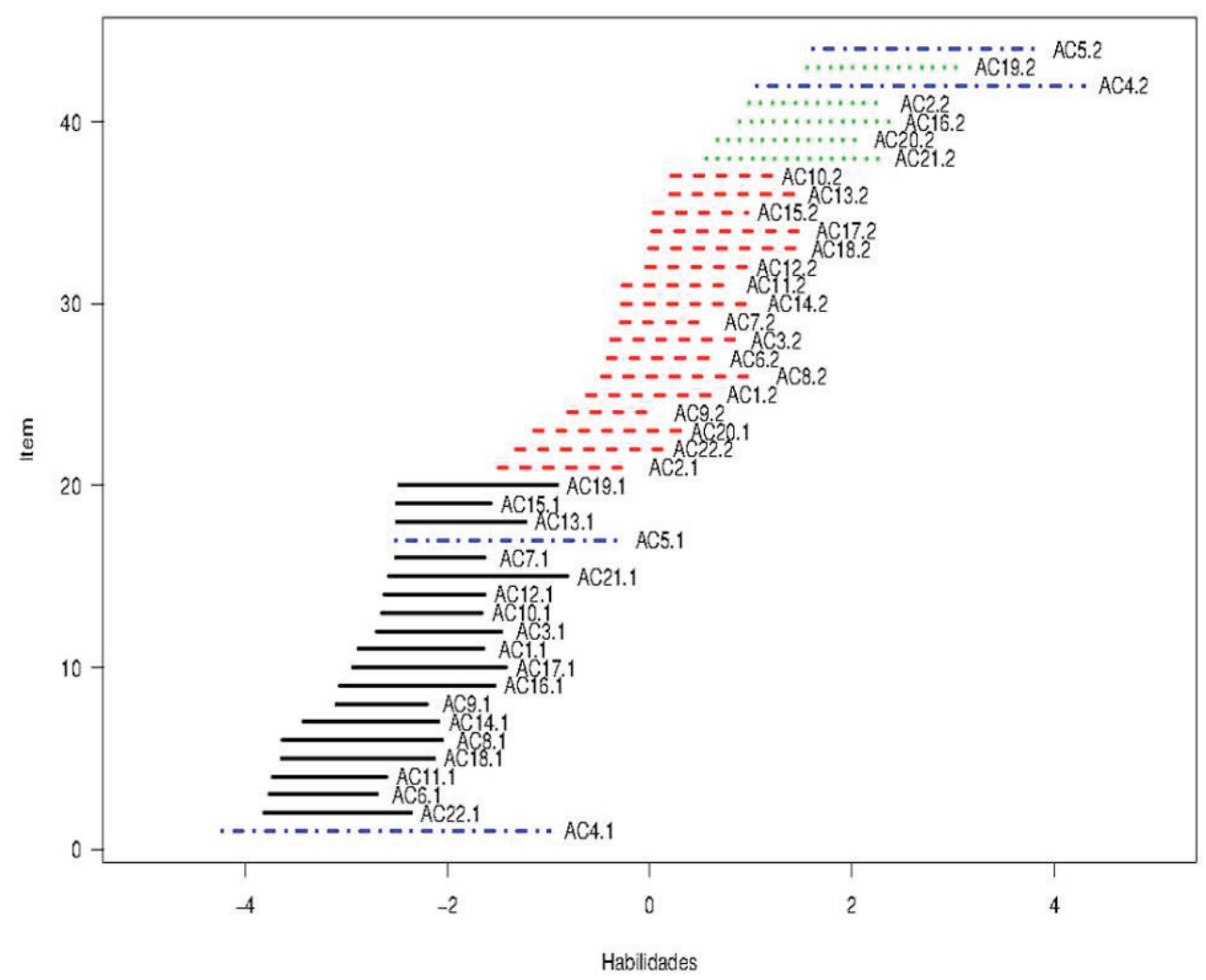

Figura 3. Intervalos por item para el anclaje de la escala.

Fuente: elaboración propia

nulo) no se incluyó ninguna covariable de los participantes; en el segundo (M1) se incorporaron las variables de estrato socioeconómico del estudiante, número de personas con las que convive, su edad y el grado que cursa; en el segundo modelo no se incluyó ninguna interacción. El estrato y el curso se incluyeron como variables ordinales categóricas mediante la trasformación de las categorías por polinomios ortogonales del grado adecuado.

La Tabla 7 presenta el promedio (con su error estándar, $\mu$ y EE respectivamente), la varianza $\left(\sigma^{2}\right)$ y la desviación estándar $(\sigma)$ residual del nivel de estudiante para los dos modelos, así como los valores de los criterios de información de Akaike (AIC) y Bayesiano (BIC); también se presenta el porcentaje de la varianza residual del nivel de estudiante explicada por la introducción de las variables señaladas. Teniendo en cuenta la magnitud del porcentaje de varianza explicada y que tanto el AIC como el BIC son menores para el modelo nulo, la comparación de los dos modelos ajustados indica que las variables de estudiante seleccionadas no permiten explicar la variabilidad del acoso escolar en los estudiantes participantes.

\section{Análisis de correspondencias múltiples y de clasificación}

En la Figura 4 se presenta el histograma de los valores propios asociados a la matriz de Burt; en ella se aprecia que predominan los 5 primeros factores de los cuales se interpretan los dos primeros a partir del primer plano factorial presentado en la Figura 5; allí se presentan las cargas de cada categoría en las dos primeras dimensiones obtenidas mediante el ACM, las categorías se representan por triángulos rojos en las figuras las siguientes convenciones: $\mathrm{N}=$ 'Nunca', AV = 'A veces' y CF = 'Con frecuencia', seguidas del número del ítem en el cuestionario; 
TABLA 7

Comparación de los modelos de regresión latente

\begin{tabular}{ccc}
\hline & \multicolumn{2}{c}{ Modelo } \\
\cline { 2 - 3 } & $\mathrm{MN}$ & $\mathrm{M} 1$ \\
\hline$\mu$ & -2.482 & -2.76 \\
$\mathrm{EE}$ & 0.045 & 0.47 \\
$\sigma$ & 2.521 & 2.515 \\
$\sigma 2$ & 6.358 & 6.328 \\
Var. Expl. (\%) & & 0.471 \\
AIC & 103619 & 103624 \\
BIC & 103638 & 103752 \\
\hline
\end{tabular}

Fuente: elaboración propia

los triángulos verdes representan la ubicación de las variables ilustrativas en el plano factorial y los triángulos grises representan a los estudiantes. El primer plano muestra la estructura evidenciada por estos primeros ejes: las categorías se agrupan y ordenan de acuerdo con la frecuencia con que ocurren las situaciones descritas por los ítems.

\section{Análisis de clasificación}

De acuerdo con el criterio propuesto por Barbary (1996), el número de factores del ACM conservados para realizar la clasificación de los individuos se determina obteniendo la clasificación variando la cantidad de factores conservados y seleccionando aquella cantidad de factores para la cual se ubique el menor número de individuos en la clase más grande. Es decir, si se conservaran cuatro factores y la clase con más individuos contuviera 200 de ellos, y si con cinco factores, la clase más grande contuviera 220 individuos, se optaría por conservar cuatro factores en vez de cinco. De acuerdo con este criterio y habiendo obtenido las clasificaciones conservando desde 1 hasta 20 factores, se seleccionó la clasificación conservando los primeros cinco factores. De acuerdo con la relación entre índices de nivel más altos asociados a esta clasificación, se seleccionaron tres clases por describir. La caracterización de la partición optimizada de cada una de las clases es:

Clase 1 (53.7\%: 1.732 individuos). Este grupo se encuentra conformado por los estudiantes que manifiestan que Nunca les ocurre cualquiera de las situaciones descritas por los ítems. Son estudiantes principalmente de $10 .$. grado, de estrato 1 y de 16 años.

Clase 2 (34.3\%: 1.107 individuos). Este grupo está formado por los estudiantes que en la mayoría de

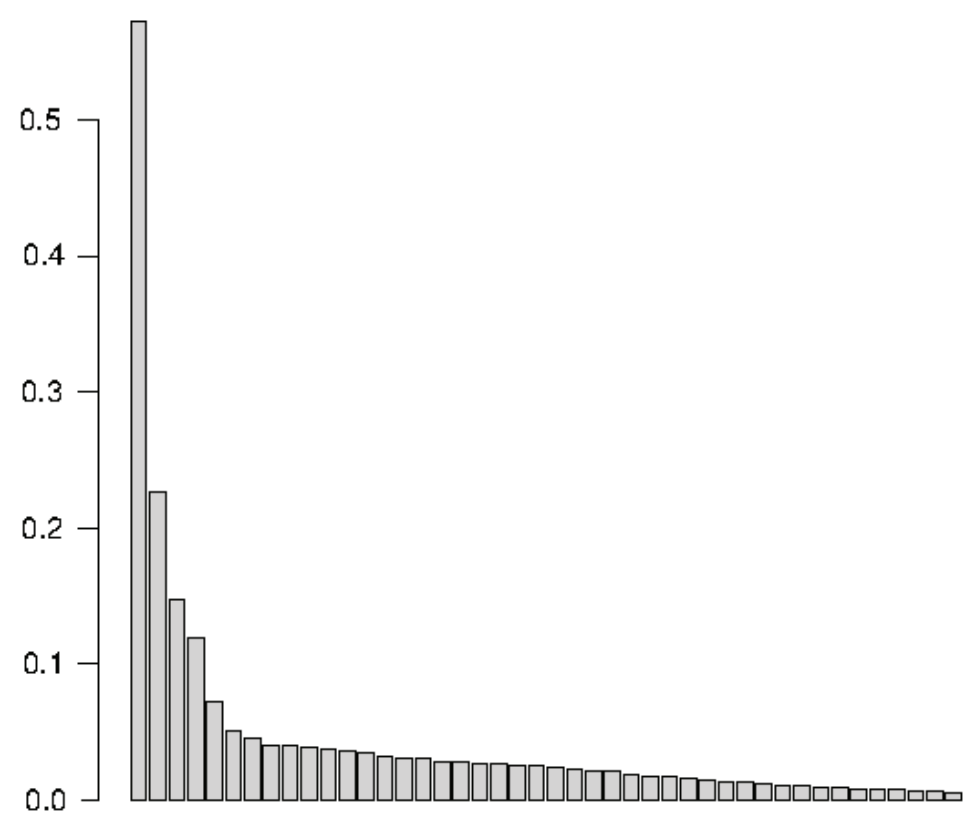

Figura 4. Histograma de los valores propios ACM

Fuente: elaboración propia

| Universitas Psychologica | V. 13 | No.2 | Abril-junio | 2014 | 


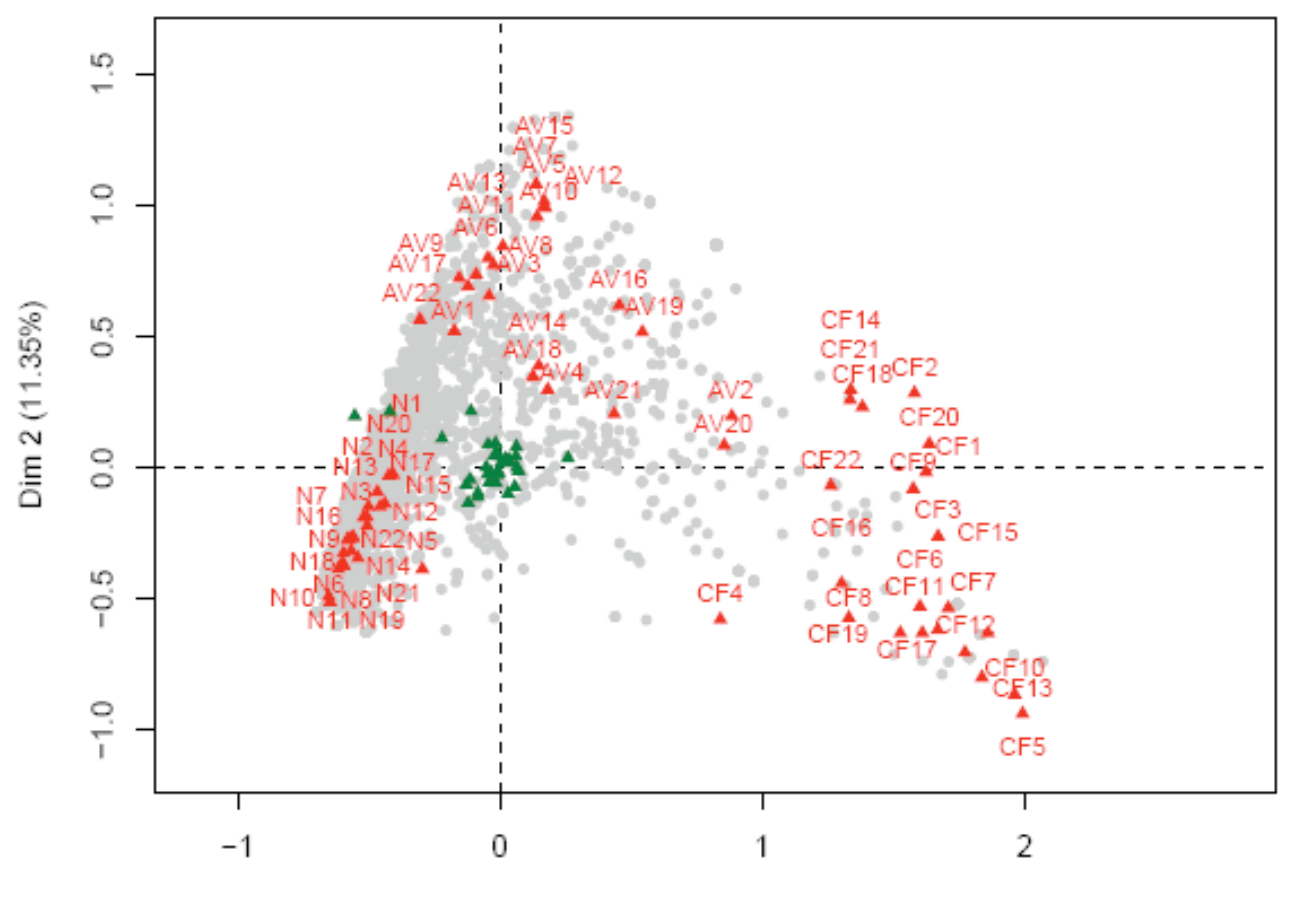

$\operatorname{Dim} 1(28.61 \%)$

Figura 5. Primer plano factorial.

Fuente: elaboración propia

las preguntas manifiestan que Nunca o A veces les ocurren la mayoría de situaciones descritas en los ítems; pero que A veces o Con frecuencia les ocurre que cambien malintencionadamente sus palabras o acciones, les interrumpan continuamente impidiéndoles expresarse, les impidan comunicarse, escondan sus objetos personales y les digan apodos que no les gustan. Además, la mayoría reporta que Nunca les ocurre que les quiten las onces. Los estudiantes en este grupo cursan especialmente

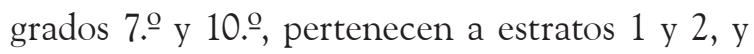
tienen 16 años.

Clase 3 (12\%: 387 individuos). Los estudiantes de este grupo reportan que Con frecuencia les ocurren la mayoría de las situaciones descritas en los ítems. En particular, reportan que $A$ veces o Con frecuencia les obligan a hacer cosas que pongan en riesgo su salud, no hablan con ellos, les interrumpan continuamente impidiéndoles expresarse, les hacen bromas crueles sobre su aspecto, dañan sus objetos personales, les quitan las onces y les inducen a actuar violentamente. Estos estudiantes tienen principalmente 14 años y pueden encontrarse en cualquier curso o estrato.

\section{Discusión}

Si bien las dos metodologías empleadas difieren con respecto a la unidad de análisis sobre la que se enfocan (los ítems en el caso de la TRI y los estudiantes en el caso del ACM y clasificación), los resultados de ambos ofrecen un panorama similar con respecto al cuestionario de acoso escolar. Por ejemplo, se puede observar que las clases de estudiantes encontradas mediante el análisis de clasificación corresponden en forma gruesa con estudiantes que, bajo la descripción de la escala obtenida mediante el GRM, se encuentran en los niveles 0,1 y 3 . En este sentido, de acuerdo con los análisis realizados, el porcentaje de estudiantes participantes sometidos a altos grados de acoso escolar resulta más bajo que el encontrado originalmente por Cepeda et al. (2008) (12\% vs. $21.8 \%)$, y es más cercano al reportado por Chaux 
et al. (2009) en grado noveno (14.7\%) a la pregunta de si el estudiante ha sido víctima de acoso escolar en los últimos dos meses; esto podría indicar que ante esta pregunta, y dada la descripción de acoso presentada en el caso utilizado por Chaux et al. (2009), los estudiantes dan respuestas afirmativas cuando se presenta la mayor magnitud de acoso observada en este estudio. Asimismo, se puede observar que el ordenamiento de las categorías de respuesta sobre el primer factor del ACM coincide bastante con el ordenamiento de los umbrales obtenidos mediante el GRM. Estos resultados sugieren que la descripción del acoso lograda a partir únicamente del puntaje en el cuestionario es suficiente para dar cuenta de las diferencias individuales de los estudiantes evaluados; en este sentido, la congruencia de los dos tipos de análisis, junto con el ajuste apropiado entre los datos y el supuesto de unidimensionalidad, evidencian que el uso de un solo índice o puntaje a partir del cuestionario es un indicador válido de la percepción que los estudiantes tienen del acoso escolar en un rol de víctimas del mismo y que puede interpretarse en términos de la magnitud del acoso que padece el estudiante.

Por otra parte, a partir del re-análisis presentado, se ha obtenido una mejor comprensión de la relación entre cada uno de los indicadores de acoso escolar que componen la escala y la magnitud del acoso de que es víctima un niño. En especial, se puede observar que algunos de los ítems son indicadores de un grado de acoso más severo que otros, así como que algunos de los ítems incluidos inicialmente no dan mucha información sobre el grado de acoso padecido por el niño. A este respecto, a partir de los resultados de este estudio, pudo definirse una escala de acoso cuyo lenguaje ha sido adaptado al uso colombiano, y para la cual se han identificado las preguntas más relevantes. La escala obtenida presenta un índice de confiabilidad alto, aspecto que ha sido señalado como de especial preocupación cuando se emplea solamente una pregunta global sobre haber padecido acoso (cf., Chaux et al., 2009), similar al de la escala original (0.962, Oñate \& Piñuel, 2005), y que es mayor a las confiabilidades reportadas para otras escalas de acoso escolar reseñadas por Hamburger et al. (2011) y por Rodríguez y Martínez (2009).

A partir de los resultados del análisis de clasificación y de las coordenadas de los estudiantes en el primer factor del ACM, se observa que pocos o ninguno de los estudiantes se encuentran en los nivel 2 y 4 descritos para la escala del GRM; en este sentido, a partir de los resultados obtenidos mediante TRI y anclaje de la escala, y en la medida en que el GRM parece ajustarse adecuadamente al cuestionario, es posible obtener descripciones sobre magnitudes de acoso que no fueron observadas en la muestra, y que para otros estudiantes, si se estimara una magnitud de acoso en los rangos correspondientes, la descripción encontrada resultará adecuada.

La principal limitación de los resultados de este estudio se encuentra en que debido a la población sobre la cual fue obtenida la muestra de estudiantes, no es posible establecer la generalizabilidad de los resultados, en especial los concernientes a los grupos de estudiantes que padecen de cierta magnitud de acoso y el porcentaje que representan, más allá de Ciudad Bolívar (Bogotá, Colombia). Si bien la similitud del porcentaje del grupo más afectado con el encontrado por Chaux et al. (2009) pareciera indicar que lo encontrado en Ciudad Bolívar es parecido al país, son necesarios otros estudios que permitan corroborar esto.

Por otra parte, las alternativas seguidas en este estudio no son las únicas formas a partir de las cuales se puede abordar el análisis del acoso. Por ejemplo, Notelaers, Einarsen, Witte y Vermunt (2006) optan por emplear un análisis de clases latentes para examinar los resultados de un cuestionario de acoso en el ámbito laboral; la aproximación por clases latentes constituye una alternativa similar al ACM por lo cual puede ser interesante una comparación entre este procedimiento y el ajuste de un modelo TRI. Los resultados de este estudio permiten establecer que el uso del cuestionario de acoso escolar con el fin de obtener un solo puntaje es válido para el contexto escolar y el cuestionario analizado, es posible que estos resultados se repliquen también para otros cuestionarios o en el contexto laboral. 


\section{Referencias}

Aitkin, M., \& Aitkin, I. (2011). Statistical modeling of the National Assessment of Educational Progress. New York: Springer.

Antal, T. (2007). On the latent regression model of Item Response Theory (Inf. téc. No. RR-07-12). Princeton, NJ: Educational Testing Service.

Barbary, O. (1996, diciembre). Una aplicación del análisis cualitativo. La tipología de trayectorias individuales. En Seminario de capacitación e investigación. Recolección análisis de datos longitudinales (pp. 111120). Bogotá: PRESTA-ORSTOM/Universidad Nacional de Colombia.

Beaton, A. E., \& Allen, N. L. (1992). Interpreting scales through scale anchoring. Journal of Educational Statistics, 17(2), 191-204.

Cepeda C., E., Pacheco Durán, P. N., García Barco, L. \& Piraquive Peña, C. J. (2008) Acoso escolae a estudiantes de educación básica y media. Revista de Salud Pública, 10(4), 517-528.

Cervantes, V. H., Cepeda C., E. \& Camargo, S. (2008) Una propuesta para la obtención de niveles de desempeño en los modelos de Teoría de Respuesta al Ítem. Avances en Medición, 6(1), 45-54.

Chaux, E., Bustamante, A., Castellanos, M., Jiménez, M., \& Mejía, M. I. (2007). KitPapaz para el manejo de la intimidación escolar. Disponible en http://www. redpapaz.org/kitpapaz/intimidacion.html

Chaux, E., Molano, A., \& Podlesky, P. (2009). Socioeconomic, socio-political and socio-emotional variables explaining school bullying: A countrywide multilevel analysis. Aggressive Behavior, 35(6), 520-529.

Chessel, D., Dufour, A. -B., \& Thioulouse, J. (2004). The ade 4 package-I- One-table methods. R News, 4(1), 5-10.

Cho, S. J., Li, F., \& Bandalos, D. (2009). Accuracy of the parallel analysis procedure with polychoric correlations. Educational and Psychological Measurement, 69(5), 748-759.

Fleming, L. C., \& Jacobsen, K. H. (2009). Bullying and symptoms of depression in Chilean middle school students. Journal of School Health, 79(3), 130-137.

Fleming, L. C., \& Jacobsen, K. H. (2010). Bullying among middle-school students in low and middle income countries. Health Promotion International, 25(1), 73-84.

Glorfeld, L. W. (1995). An improvement on Horn's parallel analysis methodology for selecting the correct number of factors to retain. Educational and Psychological Measurement, 55(3), 377-393.

Hamburger, M. E., Basile, K. C., \& Vivolo, A. M. (2011). Measuring bullying victimization, perpetration, and bystander experiences: A compendium of assessment tools. Atlanta, GA: Centers for Disease Control and Prevention, National Center for Injury Prevention and Control.

Husson, F., Josse, J., Le, S., \& Mazet, J. (2007). FactoMineR: Factor analysis and data mining with $\mathrm{R}$ [Manual de software de cómputo]. Disponible en http://factominer.free.fr

Jiménez, M., Castellanos, M., \& Chaux, E. (2009). Manejo de casos de intimidación escolar: método de preocupación compartida. Pensamiento Psicológico, 6(13), 69-86.

Kyriakides, L., Kaloyirou, C., \& Lindsay, G. (2006). An analysis of the Revised Olweus Bully/Victim Questionnaire using the Rasch measurement. British Journal of Educational Psychology, 76(4), 781-801.

Lebart, L., Piron, M., \& Morineau, A. (1995). Statistique exploratoire multidimensionelle. Paris: Dunod.

Mynard, H., \& Joseph, S. (2000). Development of the multidimensional peer-victimization scale. Aggressive Behavior, 26(2), 169-178.

Notelaers, G., Einarsen, S., Witte, H. de \& Vermunt, J. K. (2006). Measuring exposure to bullying at work: The validity and advantages of the latent class cluster approach. Work E\& Stress, 20(4), 288-301.

Oñate Cantero, A., \& Piñuel, I. (2005). Informe Cisneros VII: Violencia y acoso escolar en alumnos de primaria, ESO y bachiller. Informe preliminar (Informe técnico). Madrid: Instituto de Innovación Educativa y Desarrollo Directivo.

R Development Core Team (2008). R: A language and environment for statistical computing [Manual y software de cómputo]. Viena, Austria: R Foundation for Statistical Computing.

Rizopoulos, D. (2006). ltm: An R package for latent variable modelling and item response theory analyses. Journal of Statistical Software, 17(5), 1-25. 
Acoso en El AMBIENTE ESCOLAR: ANÁLISIS DE Un CUESTIONARIO MEDIANTE TEORÍA

De Respuesta al Ítem y AnÁlisis de Correspondencias Múltiples

Rodríguez, O. R., \& Martínez, J. G. (2009). Cinco instrumentos objetivos para medir el maltrato por abuso de poder entre pares. Avances en Medición, 7, 129-134.
Samejima, F. (Ed.). (1969). Estimation of latent ability using a response pattern of graded scores (Psychometrika Monograph Supplement № 17). New York: Psychometric Society. 


\section{Apéndice \\ Ítems que conforman el Cuestionario de Acoso \\ Escolar}

\begin{tabular}{ll}
\hline Código & Ítem \\
\hline AC1 & Me obligan a realizar cosas que no quiero \\
AC2 & Me obligan a hacer cosas que ponen en peligro mi salud a propósito \\
AC3 & Le prohíben a otros hablar conmigo \\
AC4 & No hablan conmigo \\
AC5 & Menosprecian mi trabajo, no importa lo que haga o diga \\
AC6 & Hacen críticas y reproches por cualquier cosa que haga o decisión que tome \\
AC7 & Me humillan y desprecian en público \\
AC8 & No cuentan conmigo para realizar actividades en la clase \\
AC9 & Cambian malintencionadamente lo que digo o hago \\
AC10 & Me culpan de todo lo malo que pasa \\
AC11 & Hacen burlas o bromas de mí \\
AC12 & Me amenazan de manera verbal o mediante gestos \\
AC13 & Me ignoran o me excluyen \\
AC14 & Me interrumpen continuamente impidiendo expresarme \\
AC15 & Impiden comunicarme \\
AC16 & Hacen bromas crueles acerca de mi aspecto físico \\
AC17 & Me lanzan objetos \\
AC18 & Esconden mis objetos personales \\
AC19 & Dañan mis objetos personales \\
AC20 & Me quitan las onces \\
AC21 & Me inducen a actuar violentamente \\
AC22 & Me dicen apodos que no me gustan \\
\hline
\end{tabular}

Fuente: Adaptada de la Tabla 2 de Cepeda et al. (2008) 